\title{
Sensitivity to relative disparity in early visual cortex of pigmented and albino ferrets
}

\author{
C. Kalberlah $\cdot$ C. Distler $\cdot$ K.-P. Hoffmann
}

Received: 11 June 2008 / Accepted: 6 August 2008 / Published online: 23 August 2008

(C) The Author(s) 2008. This article is published with open access at Springerlink.com

\begin{abstract}
To investigate binocular interactions as the neuronal substrate for disparity sensitivity in the ferret (Mustela putorius furo), we measured the effects of relative horizontal disparities on responses of neurons in areas 17 and 18 of the visual cortex. Stimulation by moving bars and sinusoidal gratings showed that about half of our sample in pigmented ferrets was sensitive to relative horizontal disparity. This also included many neurons, which were classified as only monocularly activated when testing either eye alone. However, the tuning width was about two or three times coarser (median tuning width $4^{\circ}$ of visual angle) than that in the cat. In albino ferrets, only $8 \%$ of the neurons in the early visual cortex displayed some sort of disparitydependent binocular interactions, but none could be clearly identified as relative disparity-coding neuron.
\end{abstract}

Keywords Mustela putorius furo · Albinism · Striate cortex $\cdot$ Disparity sensitivity

\section{Introduction}

Because of the manifold anatomical and physiological alterations in their visual system, albinos experience a number of behavioural defects (for review, see Jeffery 1997;

C. Kalberlah · C. Distler · K.-P. Hoffmann ( $\square)$

Allgemeine Zoologie und Neurobiologie,

Ruhr-Universität Bochum, Postfach 102148,

44780 Bochum, Germany

e-mail:kph@neurobiologie.rub.de

Present Address:

C. Kalberlah

Max Planck Institute for Human Cognitive and Brain Sciences,

Stephanstraße 1a, 04103 Leipzig, Germany
Hoffmann et al. 2004; Hupfeld et al. 2006). In short, due to a mutation affecting the tyrosine metabolism, the spatiotemporal maturation in the retina is altered, and the decussation pattern shows a reduction of ipsilateral projections (Morgan et al. 1987). One of the consequences is an abnormal representation of the visual field in areas 17 and 18, a reduced binocularity in visual cortex (e.g. Hubel and Wiesel 1971; Shatz 1977; Antonini et al. 1981; Akerman et al. 2003), and absence of stereopsis in the Siamese cat (Packwood and Gordon 1975). Albino strains are readily accessible in ferrets. Thus, the ferret offers a unique opportunity to study the pathology of cortical mechanisms underlying depth perception in albinos and compare it to wild-type conspecifics.

While the existence of monocular cues for depth perception was known already in ancient times and was rediscovered in the early sixteenth century primarily by Leonardo da Vinci (Howard and Rogers 1995), first behavioral and psychophysical studies of stereopsis as a result of binocular disparity were conducted by Wheatstone (1838) and extended by Julesz (1971). Neurophysiological studies by Barlow et al. (1967) and Pettigrew et al. (1968) described disparity-selective neurons in the primary visual cortex (V1) of anesthetized cats and provided first evidence for binocular disparity in V1 as a possible basis for stereopsis. Subsequent studies in alert, fixating monkeys confirmed the concept of stereopsis in the early visual areas (Poggio and Fischer 1977; Poggio et al. 1988; Cumming and Parker 1999). The exact mechanisms underlying stereopsis, however, are still unknown and have been a matter of discussion for years (e.g. DeAngelis et al. 1995; Ohzawa et al. 1996, 1997; Anzai et al. 1997; Bridge et al. 2001; Prince et al. 2002; Nienborg et al. 2004).

Four basic categories of disparity-coding neurons were established. First, neurons respond best to objects lying either in front of the horopter (crossed disparity, near-coding 
cell) or, second, very near the horopter (zero-disparity-coding cell) or, third, behind the horopter (uncrossed disparity, far-coding cell). The fourth group, the tuned-inhibitory cells, responds optimally to objects at all disparities except those near zero. Although it is argued that especially the three groups of excitatory neurons may not be discrete (LeVay and Voigt 1988; Prince et al. 2002), application of this classification appears to be beneficial for finding differences of the response profiles between categories (Poggio 1995). Thus, this classification is still commonly used, but it should be emphasized that the early visual areas code for the whole range of disparities. This led to the assumption that stereopsis is equivalent to depth perception (Barlow et al. 1967). Although this hypothesis is no longer valid (Cumming and Parker 1999), it remains that neurons in V1 perform essential preprocessing underlying stereoscopic depth perception in higher areas.

Up to now, the mechanisms of stereopsis were mainly studied in the early visual areas of mammals with frontally located eyes. By contrast, little is known about coding of retinal disparities in other species with smaller interocular distance or lower visual acuity.

We chose the ferret for our experiments, because the ferret's eyes are less frontally located and the interocular distance is smaller than that in cats or primates. Binocular visual field overlap amounts to about $80^{\circ}$ of visual angle (Garipis and Hoffmann 2003). The ferret's visual acuity is about half the visual acuity of cat (cat: Blake and Antoinetti 1976; Jacobson et al. 1976; Smith et al. 1980; ferret: Pontenagel and Schmidt 1980; Wen et al. 1985; Price and Morgan 1987; Hupfeld et al. 2006). Furthermore, ferrets are frequently used in developmental studies due to the fact that they are born at an early gestational age. Thus, while ferret and cat visual systems are very similar, early and immature developmental stages are available postnatally in ferret (Greiner and Weidman 1981). Thus, ferret is an interesting model for studying developmental and adult disparity mechanisms in a comparative way.

\section{Material and methods}

\section{Animals}

Electrophysiological recordings were performed in six pigmented and three albino adult female ferrets (Mustela putorius furo) 1-2 years of age. All experiments were approved by the local authorities (Regierungspräsidium Arnsberg) and were carried out in accordance with the Deutsche Tierschutzgesetz of 12 April 2001, the European Communities Council Directive of 24 November 1986 (S6 609 EEC), and the National Institutes of Health guidelines for care and use of animals for experimental procedures. The animals were bred and raised in the animal facility of the Department of General Zoology and Neurobiology, Ruhr Universitaet Bochum, and were group-housed in an enriched environment with access to an outdoor enclosure.

\section{Surgery}

Animals were initially treated with $0.05 \mathrm{mg}$ atropine sulphate (Braun) i.m. and anesthetized with $20 \mathrm{mg} / \mathrm{kg}$ ketamin $\left(\right.$ Ketavet $^{\circledR}$ ) and $2 \mathrm{mg} / \mathrm{kg}$ xylacine $\left(\right.$ Rompun $\left.{ }^{\circledR}\right)$ i.m. They were intubated through the mouth, and a catheter was introduced into the cephalic vein. After additional local anesthesia with bupivacain hydrochloride, animals were placed into the stereotactic frame and artificially ventilated with air containing $0.2-0.6 \%$ halothan as needed throughout the entire experiment. Heart rate, endtidal $\mathrm{CO}_{2}$, and body temperature were monitored and maintained at physiological levels. The depth of anesthesia was controlled based on the heart rate and cardiac reaction to tactile stimuli. Pupils were dilated with tropicamide (Mydriaticum ${ }^{\circledR}$ Stulln). Corneae were protected with contact lenses chosen with a refractometer (Rodenstock) to correct the optics of the eyes to the distance of the stimulation monitors (see "Visual stimulation"). The skin overlying the skull was cut, the temporalis muscle was deflected, and a craniotomy was performed allowing access to areas 17 and 18 of visual cortex. Additionally, a head post was implanted, by which the animals were held during the recording. During the recordings, the animals were paralyzed with alcuronium chloride $\left(\right.$ Alloferin ${ }^{\circledR}$ ) by an initial i.v. injection of $0.1 \mathrm{mg} / \mathrm{kg}$ and an i.v. infusion of $0.1 \mathrm{mg} / \mathrm{kg}$ per hour. After completion of the recording sessions, the wound was closed in appropriate layers and covered with antibiotic ointment $\left(\right.$ Nebacetin $\left.{ }^{\circledR}\right)$. After full recovery, the animals were returned to their home enclosure and treated with analgetics (Carprofen, Rimadyl ${ }^{\circledR}$ ) for 2 days and broadband antibiotics (Enrofloxacin, Baytril $^{\circledR}$ ) for 1 week after surgery. Electrophysiological recordings were repeated three to five times with 3 weeks recovery in-between. Because the quality of recordings during longterm acute experiments turned out to decline with time, we instead chose repeated shorter recording sessions (12-13 h duration). With this method, the quality and the amount of data recorded from individual animals dramatically increased. The procedure was well tolerated by the animals as shown by their normal feeding and play behavior.

\section{Electrophysiology}

Electrophysiological recordings in visual areas 17 and 18 were performed with tungsten in glass microelectrodes. To achieve penetrations from the mediodorsal to the lateroventral parts of the areas, the electrode was angled $45^{\circ}$ from 
medial to lateral in the coronal plane. Histological reconstructions of the recording sites indicate that our recordings sampled all cortical layers. Neuronal activity was conventionally amplified, passed through a window discriminator and stored for offline analysis.

Visual stimulation

To present stimuli in different horizontal disparities, we used an adapted mirror stereoscope (Ohzawa and Freeman 1986a, b). At each side of the animal, a screen (Eizo ${ }^{\circledR}$, FlexScan F57, $60 \mathrm{~Hz}$ ) was positioned at a viewing distance of $28 \mathrm{~cm}$. Thus, each screen covered about $60^{\circ}$ by $40^{\circ}$ of the visual field. "Cortex" software (Laboratory of Neuropsychology, NIMH, Bethesda, USA) was used to control visual stimulation and data acquisition. Position and extent of monocular receptive fields of recorded neurons were determined by bright bars of various sizes moved and rotated on dark background manually by means of the computer mouse. Cells were classified based on their responses as belonging to areas 17 or 18 . This electrophysiological classification of recorded neurons was applied to the further data analysis.

Two different stimuli were used. The first stimulus was a moving bright bar on dark background (size $1^{\circ}$ by $20^{\circ}$ of visual angle; moving in eight directions in $45^{\circ}$ steps; speed $5,10,20,50$, and $100 \%$; Michelson contrasts of 0.032 , $0.063,0.091,0.189,0.375,0.559,0.756$, and 0.976; background luminance $0.8 \mathrm{Cd} / \mathrm{m}^{2}$ ). The second stimulus consisted of a cyclic luminance modulated grayscale sinusoidal grating (diameter $40^{\circ}$ of visual angle; moving in eight directions in $45^{\circ}$ steps; spatial frequencies of $0.05,0.07$, $0.1,0.2,1$, and $2 \mathrm{cpd}$; temporal frequency of $1 \mathrm{~Hz}$; Michelson contrasts of $0.016,0.040,0.063,0.133,0.298,0.465$, 0.778, and 0.976; mean luminance $19.1 \mathrm{Cd} / \mathrm{m}^{2}$ ).

Each stimulus presentation was preceded and followed by a $500 \mathrm{~ms}$ presentation of the dark or gray background, respectively. Neuronal responses for different stimulus parameters were recorded separately using a pseudorandomized block design with ten repetitions per condition. Great care was taken to always cover the whole receptive field with our stimuli. These short tuning programs allowed us to determine the stimulus parameters that led to a clear activation of each recorded cell.

Using the stimulus with optimal parameters (like orientation, size, contrast, and speed), the horizontal disparity was then altered by computer-controlled displacement of the left screen in horizontal direction. Only neurons with vertical or oblique preferred orientations (up to $45^{\circ}$ from vertical) were included in this analysis. Thus, irrespective of the preferred orientation (vertical or oblique) horizontal relative disparities tested were the same. Each run consisted of increasing and decreasing values of horizontal disparity, i.e. a sequence of increasing disparities was followed by a series of decreasing disparities to exclude long-term drifts (bars: nine conditions with steps of $2^{\circ}$, or, when neurons had smaller receptive fields, with steps of $1^{\circ}, 0.5^{\circ}$, or $0.25^{\circ}$ horizontal disparity; gratings: 12 conditions with steps of $30^{\circ}$ over a full range of $360^{\circ}$ between the two dichoptically presented gratings. Thus, absolute steps of horizontal disparity depended on the spatial frequency of the grating.) Each condition was repeated ten times.

\section{Quantitative analysis}

Data were analyzed with MatLab ${ }^{\circledR}$ (Version 7.0.1, The MathWorks Inc.) using the Curve Fitting Toolbox (Version 1.1.2). Spike trains were convolved with a normalized, symmetric triangle kernel function $30 \mathrm{~ms}$ in width (Nawrot et al. 1999).

For bar stimuli, there was a clear receptive field-dependent increase and decrease of neuronal activity per sweep. We fitted the density function with a Gaussian distribution to define an analysis window of \pm one standard deviation of the Gaussian. Within that interval, we calculated the mean neuronal activity in spikes/s for each condition and not the total number of spikes in the response peaks to eliminate any bias due to different widths of the peaks with changing relative disparities.

For grating stimuli, we classified simple and complex cells via a Fourier analysis of the neuronal activity, calculating the $F_{1} / F_{0}$ ratio between the amplitude of the first harmonic of the response and the mean activity (Movshon et al. 1978; Dean and Tolhurst 1983; Ohzawa and Freeman 1986a, b; Skottun et al. 1991; but see Mechler and Ringach 2002). Neurons with highly modulated activity (relative modulation $>1$ ) were classified as simple, others as complex. For the subsequent analysis of simple cells, the amplitude of the first harmonic was used; otherwise, the mean activity was used.

Because of no clear landmarks on the retina, we could not define the eye's center of gaze. Following the assumption that differences of monocular receptive field locations in early visual areas converge to zero for aligned eyes (e.g. Bishop 1973), or converge to a certain value for unaligned eyes that represents the deviation of gaze in a given experiment, we defined the mean difference of monocular receptive field locations on both screens for each experiment (on average data from 14 neurons per experiment) as zero disparity and related the positions of the stimulus presentation to this defined zero disparity. We thus treat our disparity settings as relative disparity centered on zero as defined above. The spatial stability of the response through the right eye for which the monitor was never shifted served as a control for the lack of drifts of this eye during the recording of one neuron. In fact, we never observed such shifts 
throughout the experiment under the immobilization conditions we imposed. Only $2 \mathrm{~h}$ (minimum) after discontinuing the alloferin infusion and the recording did visible spontaneous movements of the eyes start.

To analyze a neuron's sensitivity for horizontal relative disparity, we screened our data for runs with at least one dataset of a certain horizontal disparity in which at least one measured value exceeded the range of the 2.5 -fold standard deviation of that group. These particular runs were excluded from further analysis because of possible unstable recording. We applied a nonparametric one-way ANOVA (Kruskal-Wallis, $P<0.05$ ) to screen for significant differences of mean neuronal activities at different horizontal disparities. Furthermore, multiple comparison was used to find statistically different activations in maxima or minima of relative disparity tuning curves, respectively. The difference between two disparities leading to statistically different activations was defined as the minimal difference affecting activity (MDAA). This approach leads to a statistical determination of tuning width. For better comparison with other studies, we additionally fitted disparity tuning curves with a Gaussian.

\section{Histology}

After completion of the electrophysiological experiments, the animals were euthanized with an overdose of pentobarbital and perfused transcardially with $0.9 \%$ saline and $4 \%$ paraformaldehyde-lysine-periodate. After appropriate cryoprotection, frozen frontal sections were cut at 40 or $50 \mu \mathrm{m}$, respectively, and stained for Nissl, myeloarchitecture (Gallyas 1979, as modified by Hess and Merker 1983), and cytochrome C histochemistry (Wong-Riley 1979). Recording sites were reconstructed based on microlesions, the penetration scheme and the depth reading on the electrode microdrive.

\section{Results}

\section{Recording sites}

Based on the histological reconstruction of microlesions and penetration tracks, all our recording sites were situated in either area 17 or area 18 close to the area $17 / 18$ border. This was in good agreement with our "online" electrophysiological classification of recorded cells as area 17 or 18 neurons.

Sensitivity to horizontal relative disparity in pigmented ferret-stimulation with bars

To determine a neuron's relative disparity sensitivity, the cell was first monocularly stimulated and neuronal activity was recorded to define the analysis window as one standard deviation width of the Gaussian function that was fitted to the mean density of the neuronal response during monocular stimulation (see methods, "Quantitative analysis"). Stimulus parameters were chosen to optimize the neuronal response. Based on the monocular responses, we determined the ocular dominance (OD) index and defined seven OD groups (Hubel and Wiesel 1962), which are plotted in Fig. 1. Astonishingly, the largest group comprising more than $20 \%$ of the neurons were driven by the contralateral eye only. Then, the neuronal activity was recorded during binocular stimulation with varying horizontal disparity of the stimulus. The neuronal activity was analyzed within the previously determined analysis window.

Figure $2 \mathrm{a}-\mathrm{d}$ shows peristimulus-time-histograms of the neuronal activity to exemplify relative disparity sensitive neurons of different categories stimulated by moving bars. During the disparity test, the position of the left screen was varied with respect to the left eye to achieve variation of horizontal disparity of the binocular stimulus. Thus, the position of the stimulating bar in the visual field changed for different conditions with respect to the left eye. In cases where the left eye yielded the dominant input to the cell recorded, the neuronal response directly revealed the spatial shift of the stimulus (Fig. 2c, d). In cases where the right eye yielded the dominant input, the response peak stayed spatially constant, which was taken as evidence for no residual eye movements.

Sensitivity for horizontal relative disparity can also be judged by the modulation of the neuronal activity in response to a change in horizontal disparity. Thus, to analyze a neuron's sensitivity for relative disparity, we compared the mean neuronal activity within the analysis window (peak activity instead of taking the response over the whole response period) during several trials of monocular and binocular stimulation (indicated by gray horizontal lines in Fig. 2a-d). The neurons' mean activities as a function of horizontal relative disparity are plotted in Fig. 2e-h and compared to the neuronal activities during monocular stimulation (white bars). Although two of the four cells (Fig. 2e, g) were clearly monocularly dominated, all cells were significantly modulated by variation of the horizontal disparity of binocularly presented visual stimuli.

The relative disparity-sensitive neurons were classified according to the disparities leading to their maximal neuronal activities. Cells with maximal activity at negative (crossed) horizontal disparity respond best to stimuli in front of the horopter; cells, which preferred positive (uncrossed) horizontal disparity, respond best to stimuli behind the horopter. Cells classified as near zero-disparity 

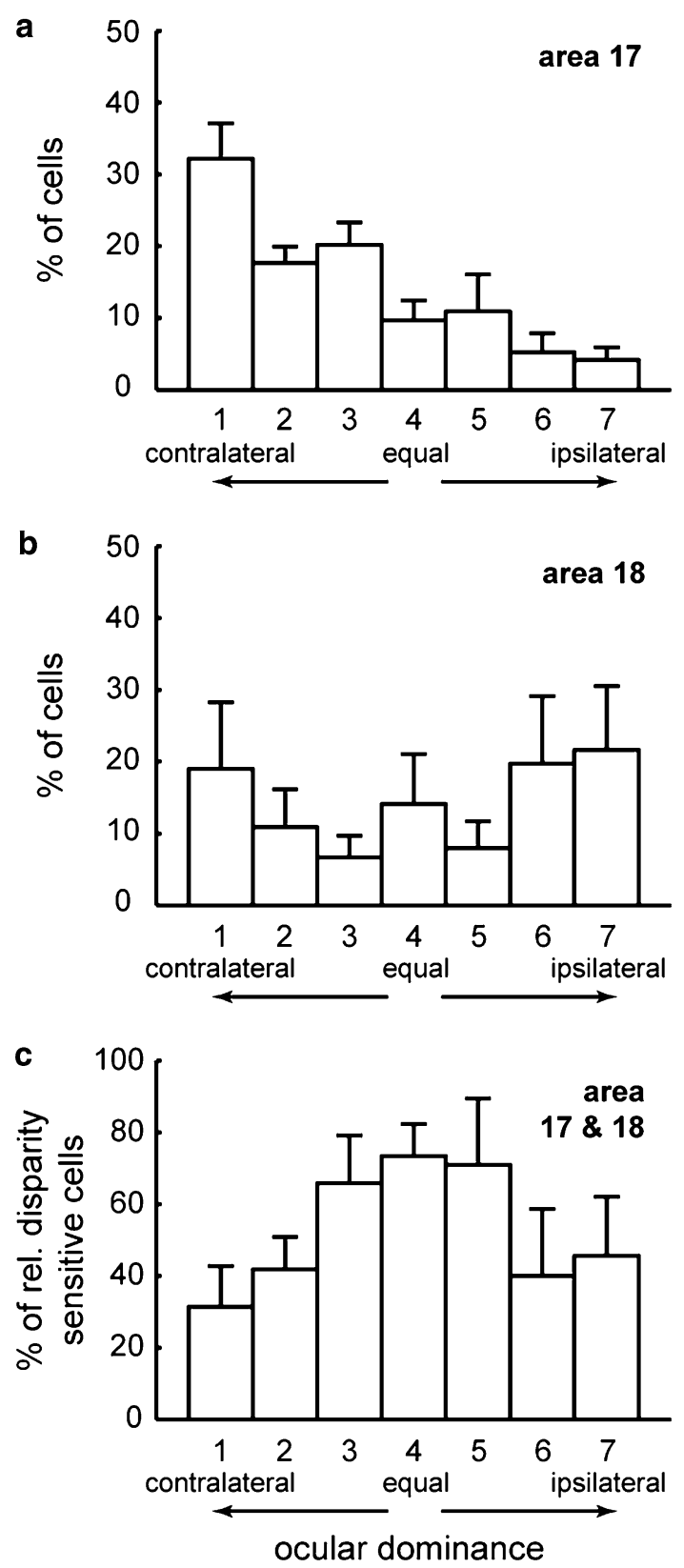

Fig. 1 Frequency histograms of ocular dominance (OD) classes. OD was calculated by (ipsilateral - spontaneous activity)/[(ipsilateral spontaneous $)+($ contralateral - spontaneous activity)]. Seven OD classes were formed by binning ODs with class boundaries at $0.0,0.1$, $0.25,0.4,0.6,0.75,0.9$, and 1.0 . a area $17, \mathbf{b}$ area 18 , abscissa OD classes from 1 (driven only by the contralateral eye) to 7 (driven only by the ipsilateral eye), ordinate average percentage of cells per animal and standard error. c Average percentage of relative disparity-sensitive cells in each OD class per animal and standard error (ordinate); abscissa as in $\mathbf{a}$ and $\mathbf{b}$

coding respond best to stimuli near the horopter. By contrast, tuned inhibitory cells show minimal activity near the horopter (Fig. 2e-h).
Sensitivity to horizontal relative disparity in pigmented ferret-stimulation with sinusoidal gratings

Figure 3a shows the neuronal activity of a relative disparity-sensitive neuron stimulated with a sinusoidal grating. Both eyes were equally effective during monocular stimulation (Fig. 3b). Varying the horizontal disparity of the presented stimulus and thus shifting one of the dichoptically presented stimuli in 12 steps of $30^{\circ}\left(360^{\circ}\right.$ corresponds to a shift of a whole sinusoidal cycle) in this neuron led to a clear modulation of the periodical response with a temporal peak difference of about $1,000 \mathrm{~ms}$, which clearly reflects the temporal frequency of the presented grating of $1 \mathrm{~Hz}$. The duration of the stimulus presentation was set to $3,000 \mathrm{~ms}$; stimulus onset is indicated by a solid, stimulus offset by a dotted vertical line in Fig. 3a.

By shifting the phase of one of the presented stimuli, the neuronal response was substantially modulated and nearly totally suppressed for a phase shift of $210^{\circ}$ (Fig. 3a). Fourier transformation of the density functions of the neuronal activity provided the amplitude of the $\mathrm{f} 1$ component at $1 \mathrm{~Hz}$ of each trial. The mean $\mathrm{f} 1$ components are plotted in Fig. $3 \mathrm{~b}$ as a function of horizontal disparity. There is a clear correlation between the periodic organization of the neuronal activity and the horizontal disparity within one whole shift of the sinusoidal grating.

Overall sensitivity to horizontal relative disparity in pigmented ferrets

Altogether, 184 cortical neurons were recorded in pigmented ferrets during stimulation with moving bars; 126 of these were classified as area 17 neurons, 47 as area 18 neurons, and 11 neurons could not be reliably classified. Of the 103 neurons $(56 \%)$ significantly modulated by horizontal disparity, $68(66 \%)$ came from area 17, 29 (28\%) came from area 18 , and $6(6 \%)$ remained unclassified. In other words, $54 \%$ of the tested area 17 neurons, $62 \%$ of the area 18 neurons, and $55 \%$ of the unclassified neurons exhibited relative disparity-dependent activity.

During stimulation with sinusoidal gratings, 27 neurons were recorded, 20 in area 17,4 in area 18 , and 3 could not be unequivocally classified. Altogether 8 neurons $(30 \%)$ were significantly modulated by horizontal disparity, 6 from area 17, 1 from area 18, and 1 unclassified. The disparity tuning curves of these relative disparity-sensitive neurons show single and clear maxima of activity at their preferred disparity. Because of the rather limited amount of data with sinusoidal stimulation, the following analysis is based on data from the bar stimulation. 


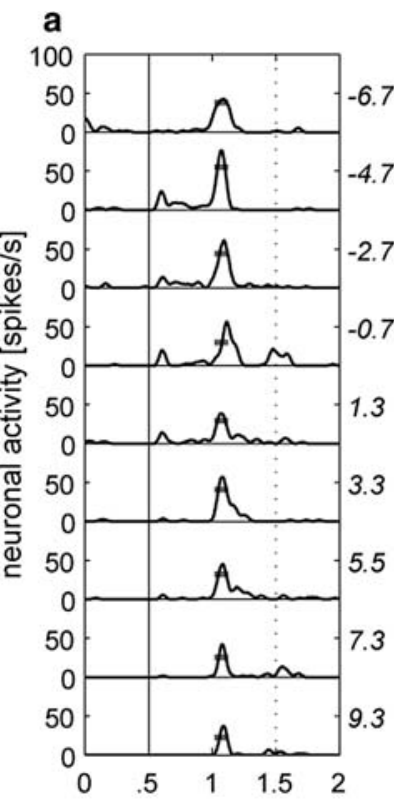

crossed

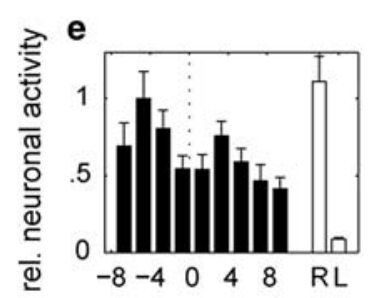

b

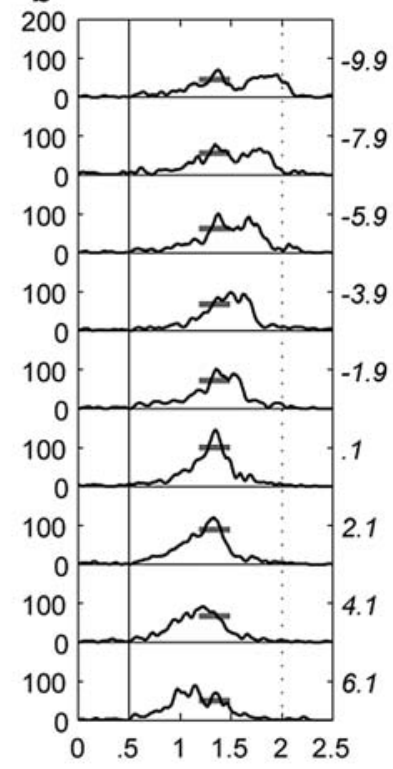

near zero

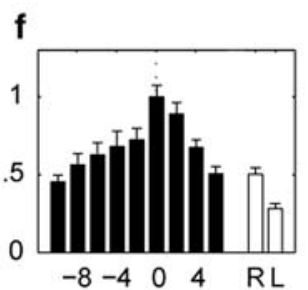

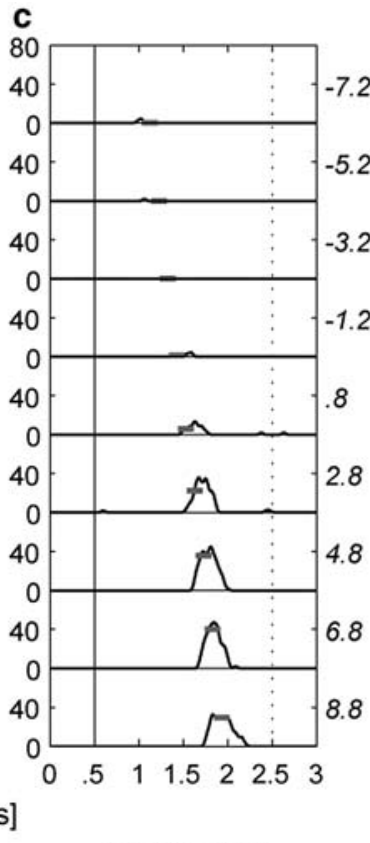

uncrossed

\section{g}

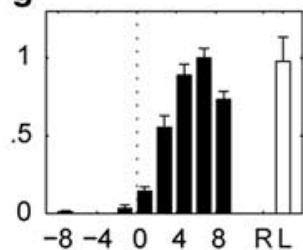

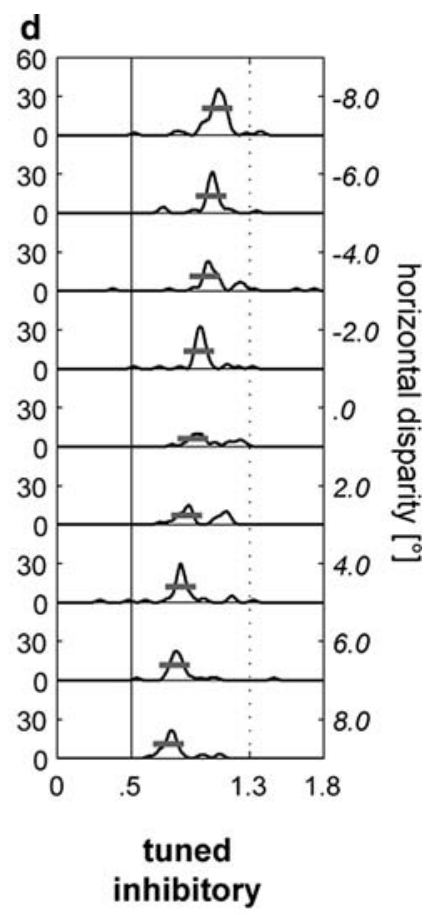

h

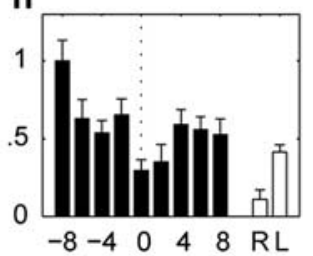

relative horizontal disparity $\left[{ }^{\circ}\right]$

Fig. 2 Examples of disparity-sensitive neurons stimulated binocularly with a moving bar. a, e crossed-, b, f near zero-, $\mathbf{c}, \mathbf{g}$ uncrossed-, $\mathbf{d}, \mathbf{h}$ tuned inhibitory cells. a-d Mean densities of neuronal activity during variation of horizontal disparity. Onset of the stimulus is marked by a continuous, offset by a dotted vertical line. Gray horizontal lines mark the analysis window; their distance from baseline indicates the mean activity within this interval. Ordinate on the left Neuronal activity

Altogether $86(84 \%)$ of the 103 disparity-sensitive neurons in pigmented ferrets stimulated with moving bars could be categorized as belonging to one of the four groups defined above. Only two tuned inhibitory cells were found (one in area 17, one in area 18). Twenty-one neurons (13 in area 17, 7 in area 18, and 1 unclassified) were near zero-disparity-coding with best responses to differences of disparity $<1^{\circ}, 28$ neurons ( 21 in area 17, 4 in area 18, and 3 unclassified) preferred crossed relative disparities, and 37 neurons (21 in area 17, 14 in area 18, and 2 unclassified) preferred uncrossed relative disparities (Fig. 4).

However, as demonstrated in Fig. 4, the distribution of preferred horizontal disparities was continuous rather than consisting of well separated categories. All relative disparity-sensitive cells show a single maximum of activation at their preferred disparity (Fig. 5). To allow comparison of (spikes/s); on the right horizontal relative disparity (degrees of visual angle, values written in italic), respectively, abscissa time in s. e-h Mean neuronal activities (ordinate) of the neurons shown in a-d depending on horizontal relative disparity (abscissa). Zero disparity is marked by the vertical dotted line. Error bars indicate one standard error. $R$ and $L$ Activity during monocular stimulation of the contralateral (right) and ipsilateral (left) eye, respectively

the tuning shapes, the tuning curves were superimposed by shifting them with an offset equal to the preferred relative crossed - and uncrossed disparity of each neuron (Fig. 5a, $\mathrm{b}, \mathrm{e}, \mathrm{f})$. The same was done for relative disparity-sensitive cells tested by sinusoidal gratings (Fig. 6). Inconclusively, we could not find any influence of preferred disparities on the response profile of relative disparity-sensitive neurons in ferret.

As described above, we based our definition of relative disparity sensitivity on the significant modulation of neuronal activity during variation of horizontal disparity of the presented stimuli. This approach directly enabled us to define a tuning width based on statistical criterions. To this end, we determined the minimal horizontal disparity that affected neuronal activity (MDAA) of all relative disparitycoding neurons. There was no significant difference in the 

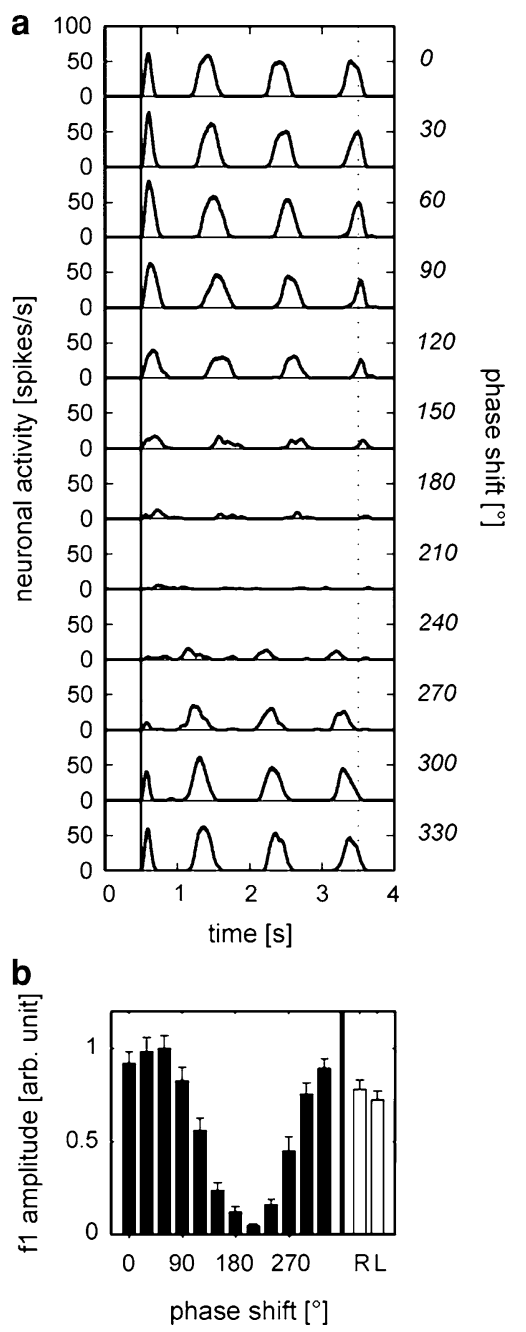

Fig. 3 Relative disparity-sensitive neuron stimulated binocularly with a moving sinusoidal grating. a Mean densities of neuronal activity during variation of horizontal disparity. Ordinate (left) neuronal activity (spikes/s), (right) phase shift in degrees, abscissa time (ms). b Amplitude of the f1 component (ordinate) depending on phase shift (abscis$s a$ ) of the neuron shown in a. For other conventions, see Fig. 2

accuracy in detecting horizontal disparities between area 17 and 18 neurons. The overall distribution of MDAAs had its median at $4.0^{\circ}$ (quartiles: $Q_{25}=3.5$ and $Q_{75}=7.0$ ).

To compare horizontal relative disparity coding in ferret with studies of other species in the literature, we averaged tuning curves of all relative disparity-sensitive neurons in pigmented ferrets and fitted a Gaussian function (Fig. 7). The half width at half height (HWHH) was $3.95^{\circ}$, corresponding exactly to the value of the MDAA median.

Sensitivity to horizontal relative disparity in albino ferrets

In albino ferrets, 98 cells were tested for relative disparity sensitivity. Of these, 62 cells were stimulated with moving bars and 36 cells were tested with sinusoidal gratings. None was activated by the ipsilateral eye alone. In strong contrast

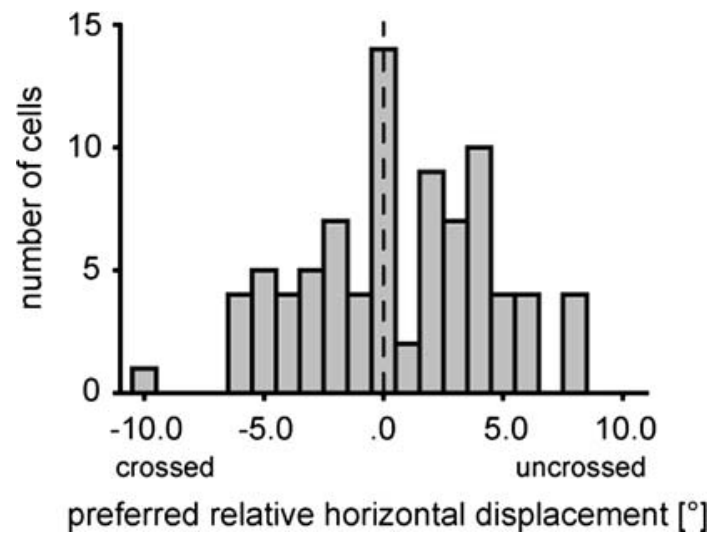

Fig. 4 Frequency histograms of preferred relative horizontal disparities (abscissa) of relative disparity-sensitive neurons binocularly stimulated with moving bars. Ordinate number of cells

to pigmented ferrets, none of the cells stimulated with sinusoidal gratings and only eight cells $(8.2 \%)$ stimulated with moving bars showed significant disparity or in the case of sinusoidal gratings phase-dependent modulation of their neuronal activity.

Furthermore, the neuronal modulation of these eight cells remained unclear. Especially, a classification into any of the stereopsis classes was impossible because of multiple local maxima or minima of the activation profile. Thus, none of the recorded cells of albino ferrets could be classified as "relative disparity-sensitive" in a narrower sense.

\section{Discussion}

Classification of primary and secondary visual cortex

We classified visual neurons "online" as recorded in areas 17 or 18 by investigating their neuronal responses to stimulation with moving bars. This classification was mainly based on the neuronal selectivity for stimulus velocity and the location of receptive fields of neighboring penetrations with respect to the representation of the vertical meridian. Since the vertical meridian is represented at the area 17/18 border, successive posterio-anterior penetrations will show a reversal of receptive fields once the border between the areas is crossed. This can be used to reconstruct the area 17/ 18 border. The electrophysiological categorization was in good agreement with the histological reconstruction in Nissl, myelin, or cytochrome oxidase-stained sections (Rockland 1985; Law et al. 1988).

Relative disparity sensitivity in pigmented ferret

To date, no behavioural data are available on stereopsis in ferrets. In the present experiments, we were able to demonstrate the presence of a possible neuronal substrate for $3 \mathrm{D}$ 
Fig. 5 Disparity-tuning curves of relative disparity-sensitive neurons stimulated with moving bars. a, b Crossed disparity-coding neurons, $\mathbf{c}, \mathbf{d}$ near zero-disparity-coding neurons, $\mathbf{e}, \mathbf{f}$ uncrossed disparity-coding neurons. a, c, e area 17 neurons, $\mathbf{b}$, d, $\mathbf{f}$ area 18 neurons. Preferred relative disparity values are shifted to zero (offset) to facilitate comparison of the tuning shape. Ordinate relative neuronal activity, abscissa relative horizontal disparity minus preferred relative disparity in degrees of visual angle area 17

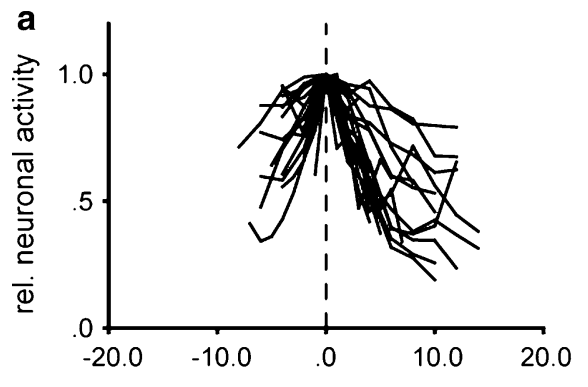

relative horizontal disparity - preferred relative disparity $\left[{ }^{\circ}\right]$
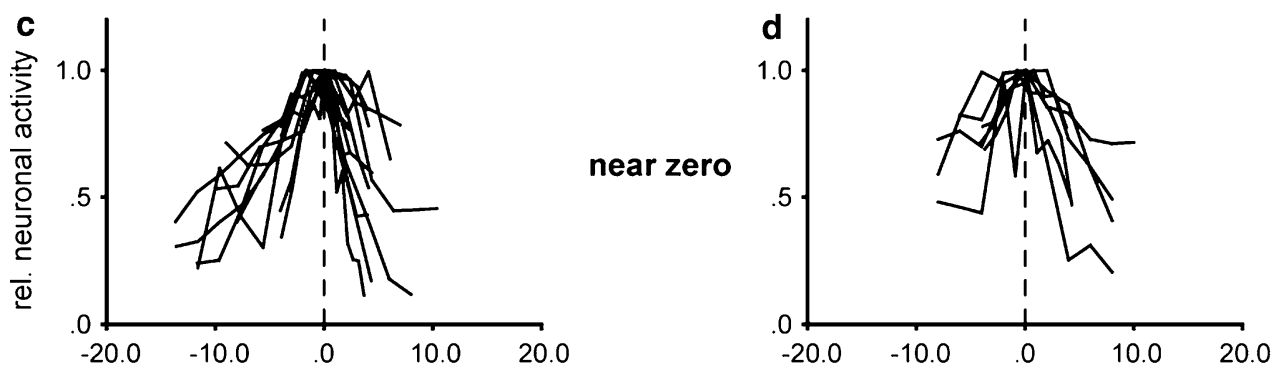

relative horizontal disparity $\left[{ }^{\circ}\right]$
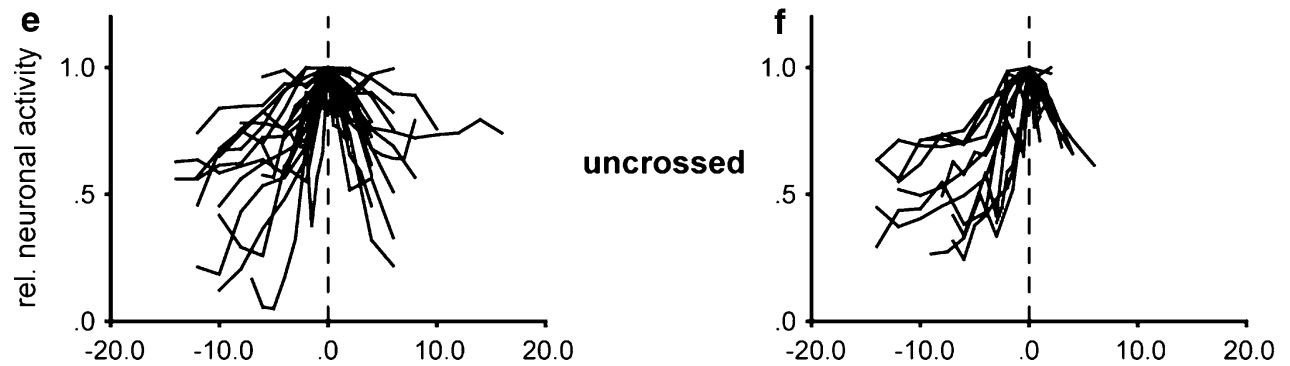

relative horizontal disparity - preferred relative disparity $\left[{ }^{\circ}\right]$

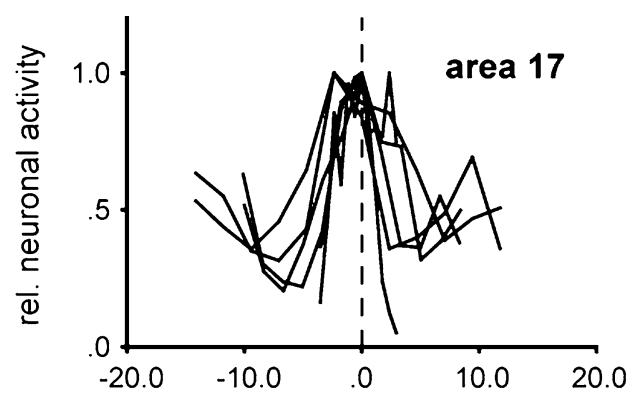

relative horizontal disparity - preferred relative disparity $\left[{ }^{\circ}\right]$

Fig. 6 Disparity-tuning curves of relative disparity-sensitive neurons in area 17 stimulated with sinusoidal gratings. Conventions as in Fig. 5

vision, i.e. neurons that preferred different horizontal disparities similar to neurons previously described in cat (Barlow et al. 1967; Pettigrew et al. 1968; DeAngelis et al. 1995; Ohzawa et al. 1996, 1997) and in monkey (Poggio and Fischer 1977; Poggio et al. 1988; Bridge et al. 2001;

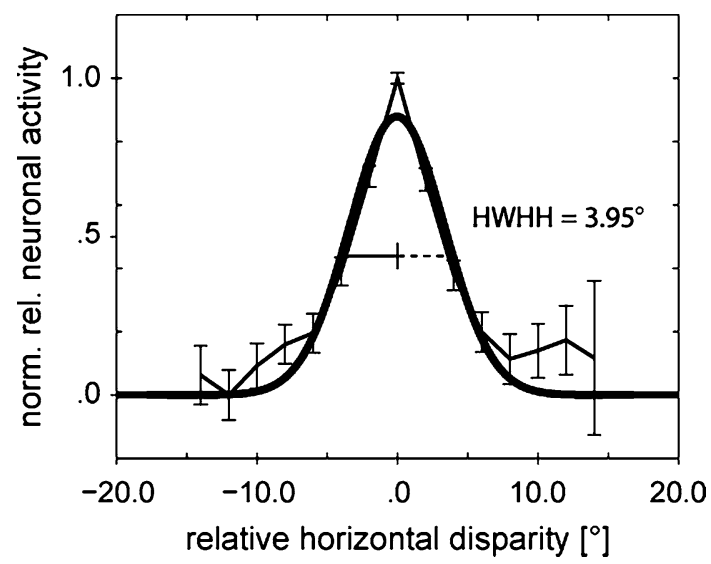

Fig. 7 Mean \pm standard error of relative neuronal activities of tuning curves collapsed from all relative disparity-sensitive neurons recorded in this study, normalized to the range from 0 to 1 (ordinate) and fitted with a Gaussian. The Gaussian has a half width at half height (HWHH) of $3.95^{\circ}$ of visual angle. Abscissa horizontal relative disparity in degrees of visual angle 
Prince et al. 2002). We modulated horizontal disparity first in coarse, then in finer steps of $2^{\circ}, 1^{\circ}, 0.5^{\circ}$, and $0.25^{\circ}$. With this procedure, we obtained tuning curves comparable to those described in cat and monkey.

Analyzing the data by quantifying the minimal disparity that affects neuronal activity (MDAA) yielded a measure for statistically significant modulations of neuronal activity, whereas techniques like fitting the tuning curves with a Gaussian and calculating the half width at half height do not indicate statistically significant modulation per se. We obtained a distribution of MDAA ranging from $1^{\circ}$ to $14^{\circ}$ with a median at $4.0^{\circ}$ of visual angle. No clear difference of MDAA between areas 17 and 18 was found, although one might expect a correlation of receptive field size and sharpness of horizontal relative disparity tuning (Nienborg et al. 2004). However, this may be due to the fact that the receptive fields in areas 17 and 18 of ferrets seem to be quite variable and overlapping in size (Manger et al. 2002).

To compare the tuning width of neurons sensitive to modulation of horizontal disparity in ferret with other species, we additionally applied a Gaussian fit to the mean tuning curve of all relative disparity-sensitive neurons. The data are well fitted by the Gaussian with a half width at half height of $3.95^{\circ}$ of visual angle, which closely corresponds to the measure of MDAA (median at $4.0^{\circ}$ ). In cat, relative disparity tunings with half widths at half height of $0.5^{\circ}-3.5^{\circ}$ and a median at $1.7^{\circ}$ of visual angle were described (LeVay and Voigt 1988; see also Pettigrew et al. 1968; Bishop et al. 1971). Though the optical quality of the ferret's eye remains unknown, other comparative parameters of ferret's and cat's visual system are well studied and can be considered to match relative disparity coding between these species. Studies of the densityand distribution of ganglion cells in ferrets (Henderson 1985) and cats (Stone 1965; Stone et al. 1978; Hughes 1975; Williams et al. 1993) and behavioral and electrophysiological studies investigating visual acuity (cat: Blake and Antoinetti 1976; Smith et al. 1980; but Jacobsen et al. 1976; ferret: Pontenagel and Schmidt 1980; Price and Morgan 1987; Hupfeld et al. 2006) indicate that visual acuity in ferret is about $50 \%$ lower than that of the cat. Furthermore, the geometrical aspect of interocular distance is fundamental for the generation of stereopsis. An anatomical study (Wen et al. 1985) found a roughly twofold interocular distance in the cat compared to the ferret. These findings indicate that relative disparity coding in ferret should be coarser at least by a factor of 2 compared to the cat. This is verified by our data.

\section{Relative disparity sensitivity in albino ferrets}

Because of the albino-specific misrouting of retinal axons, binocularity is strongly reduced in early visual cortical areas of albino ferrets (Akerman et al. 2003). One can hypothesize that this reduction or even loss of binocularity can be functionally compensated by feedback connections from the lateral suprasylvian cortex, which can be binocularly activated as shown in Siamese cats by Marzi et al. (1980) and Antonini et al. (1981). This binocular feedback might only be revealed by studying neuronal sensitivity to horizontal disparities. While much is known about callosal connections of early visual areas in pigmented ferret (Grigonis et al. 1992; Innocenti et al. 2002; Manger et al. 2002), comparable data in albino ferrets are still missing.

We were encouraged to study relative disparity tuning in albino ferrets despite the reduction in binocularity following the approach of Freeman and Ohzawa (1988), who showed that after short-term monocular deprivation in kittens, some residual relative disparity tuning was present in otherwise monocular neurons. Also many monocular neurons in our sample recorded in pigmented ferrets displayed clear relative disparity sensitivity. However, our data collected from albino ferrets show a clear reduction or rather absence of disparity tuning in the early visual areas. Although we identified eight neurons whose responses changed significantly when horizontal disparity was varied, the physiological relevance of these response patterns remained unclear because of multiple maxima or minima in the tuning curves. However, these local maxima are reminiscent of data from binocularly deprived cats, where the remaining visual responses in visual cortex are mostly irregular but nevertheless display binocular interactions (Ohzawa and Freeman 1988). These findings were attributed to disorganized receptive field structure. Thus, not a single relative disparity-tuned neuron was found in albino ferrets, although they were common in pigmented ferrets.

Our results indicate that the loss of binocular convergence of geniculate input is not compensated to a degree that maintenance of disparity signaling in early visual areas would be possible. It remains unclear to which degree monocular depth cues play a role in depth perception in the ferret. Behavioral experiments will have to clarify if the loss of disparity sensitivity in visual cortex leads to behavioral deficits in albino ferrets when a visual scene provides monocular depth cues.

Acknowledgements We thank H. Korbmacher and S. Krämer for technical support. The study was supported by grant SFB 509/A11 from the Deutsche Forschungsgemeinschaft.

Open Access This article is distributed under the terms of the Creative Commons Attribution Noncommercial License which permits any noncommercial use, distribution, and reproduction in any medium, provided the original author(s) and source are credited.

\section{References}

Akerman CJ, Tolhurst DJ, Morgan JE, Baker GE, Thompson ID (2003) Relay of visual information to the lateral geniculate 
nucleus and the visual cortex in albino ferrets. J Comp Neurol 461:217-235

Antonini A, Berlucchi G, Di Stefano M, Marzi CA (1981) Differences in binocular interactions between cortical areas 17 and 18 and superior colliculus of Siamese cats. J Comp Neurol 200:597-611

Anzai A, Ohzawa I, Freeman RD (1997) Neural mechanisms underlying binocular fusion and stereopsis: position vs. phase. Proc Natl Acad Sci USA 94:5438-5443

Barlow HB, Blakemore C, Pettigrew JD (1967) The neural mechanism of binocular depth discrimination. J Physiol 193:327-342

Bishop PO (1973) Neurophysiology of binocular single vision and stereopsis. In: Jung R (ed) Handbook of sensory physiology, vol VII/ 3, part A: integrative functions. Springer, Berlin, pp 255-306

Bishop PO, Henry GH, Smith CJ (1971) Binocular interaction fields of single units in the cat striate cortex. J Physiol 216:39-68

Blake R, Antoinetti DN (1976) Abnormal visual resolution in the Siamese cat. Science 194:109-110

Bridge H, Cumming BG, Parker AJ (2001) Modelling V1 neuronal responses to orientation disparity. Vis Neurosci 18:879-891

Cumming BG, Parker AJ (1999) Binocular neurons in V1 of awake monkeys are selective for absolute, not relative, disparity. J Neurosci 19:5602-5618

Dean AF, Tolhurst DJ (1983) On the distinctness of simple and complex cells in the visual cortex of the cat. J Physiol 344:305-325

DeAngelis GC, Ohzawa I, Freeman RD (1995) Neuronal mechanisms underlying stereopsis: how do simple cells in the visual cortex encode binocular disparity? Perception 24:3-31

Freeman RD, Ohzawa I (1988) Monocularly deprived cats: binocular tests of cortical cells reveal functional connections from the deprived eye. J Neurosci 8:2491-2506

Gallyas F (1979) Silver staining of myelin by means of physical development. Neurol Res 1:203-209

Garipis N, Hoffmann K-P (2003) Vieual field defects in albino ferrets (Mustela putorius furo). Vision Res 43:793-800

Greiner JV, Weidman TA (1981) Histogenesis of the ferret retina. Exp Eye Res 33:315-332

Grigonis AM, Rayos del Sol-Padua RB, Murphy EH (1992) Visual callosal projections in the adult ferret. Vis Neurosci 9:99-103

Henderson Z (1985) Distribution of ganglion cells in the retina of adult pigmented ferret. Brain Res 358:221-228

Hess DT, Merker BH (1983) Technical modifications of Gallyas' silver stain for myelin. J Neurosci Methods 8:95-97

Hoffmann KP, Garipis N, Distler C (2004) Optokinetic deficits in albino ferrets (Mustela putorius furo): a behavioral and electrophysiological study. J Neurosci 24:4061-4069

Howard IP, Rogers BJ (1995) Binocular vision and steropsis. Oxford University Press, Oxford

Hubel DH, Wiesel TN (1962) Receptive fields, binocular interaction and functional architecture in the cat's visual cortex. J Physiol 160:106-154

Hubel DH, Wiesel TN (1971) Aberrant visual projections in the siamese cat. J Physiol 218:33-62

Hughes A (1975) A quantitative analysis of the cat retinal ganglion cell topography. J Comp Neurol 163:107-128

Hupfeld D, Distler C, Hoffmann KP (2006) Motion perception deficits in albino ferrets (Mustela putorius furo). Vision Res 46:29412948

Innocenti GM, Manger PR, Masiello I, Colin I, Tettoni L (2002) Architecture and callosal connections of visual areas 17, 18, 19 and 21 in the ferret (Mustela putorius). Cereb Cortex 12:411-422

Jacobsen SG, Franklin KB, McDonald WI (1976) Visual acuity of the cat. Vision Res 16:1141-1143

Jeffery G (1997) The albino retina: an abnormality that provides insight into normal retinal development. Trends Neurosci 20:165-169

Julesz B (1971) Foundations of cyclopean perception. University of Chicago Press, Chicago
Law MI, Zahs KR, Stryker MP (1988) Organisation of primary visual cortex (area 17) in the ferret. J Comp Neurol 278:157-180

LeVay S, Voigt T (1988) Ocular dominance and disparity coding in cat visual cortex. Vis Neurosci 1:395-414

Manger PR, Kiper D, Masiello I, Murillo L, Tettoni L, Hundyadi Z, Innocenti GM (2002) The representation of the visual field in three extrastriate areas of the ferret (Mustela putorius) and the relationship of retinotopy and field boundaries to callosal connectivity. Cereb Cortex 12:423-437

Marzi CA, Antonini A, Di Stefano M, Legg CR (1980) Callosumdependent binocular interactions in the lateral suprasylvian area of Siamese cats which lack binocular neurons in areas 17 and 18 . Brain Res 197:230-235

Mechler F, Ringach DL (2002) On the classification of simple and complex cells. Vision Res 42:1017-1033

Morgan JE, Henderson Z, Thompson ID (1987) Retinal decussation patterns in pigmented and albino ferrets. Neuroscience 20:519535

Movshon JA, Thompson ID, Tolhurst DJ (1978) Spatial summation in the receptive fields of simple cells in the cat's striate cortex. J Physiol 283:53-77

Nawrot MP, Aertsen A, Rotter S (1999) Single-trial estimation of neuronal firing rates: from single-neuron spike trains to population activity. J Neurosci Methods 94:81-92

Nienborg H, Bridge H, Parker AJ, Cumming BG (2004) Receptive field size in V1 neurons limits acuity for perceiving disparity modulation. J Neurosci 24:2065-2076

Ohzawa I, Freeman RD (1986a) The binocular organization of simple cells in the cat's visual cortex. J Neurophysiol 56:221-242

Ohzawa I, Freeman RD (1986b) The binocular organization of complex cells in the cat's visual cortex. J Neurophysiol 56:243-259

Ohzawa I, Freeman RD (1988) Binocularly deprived cats: binocular tests of cortical cells show regular patterns of interaction. J Neurosci 8:2507-2516

Ohzawa I, DeAngelis GC, Freeman RD (1996) Encoding of binocular disparity by simple cells in the cat's visual cortex. J Neurophysiol 75:1779-1805

Ohzawa I, DeAngelis GC, Freeman RD (1997) Encoding of binocular disparity by complex cells in the cat's visual cortex. J Neurophysiol 77:2879-2909

Packwood J, Gordon B (1975) Stereopsis in normal domestic cat, Siamese cat, and cat raised with alternating monocular occlusion. J Neurophysiol 38:1485-1499

Pettigrew JD, Nikara T, Bishop PO (1968) Binocular interaction on single units in cat striate cortex: simultaneous stimulation by single moving slit with receptive fields in correspondence. Exp Brain Res 6:391-410

Poggio GE (1995) Mechanisms of stereopsis in monkey visual cortex. Cereb Cortex 5:193-203

Poggio GF, Fischer B (1977) Binocular interaction and depth sensitivity in striate and prestriate cortex of behaving rhesus monkey. J Neurophysiol 40:1392-1405

Poggio GF, Gonzalez F, Krause F (1988) Stereoscopic mechanisms in monkey visual cortex: binocular correlation and disparity selectivity. J Neurosci 8:4531-4550

Pontenagel T, Schmidt U (1980) Untersuchungen zur Leistungsfähigkeit des Gesichtssinnes bei Frettchen, Mustela putorius furo L. Z Saugetierkd 45:376-383

Price DJ, Morgan JE (1987) Spatial properties of neurons in the lateral geniculate nucleus of the pigmented ferret. Exp Brain Res 68:28 36

Prince SJ, Pointon AD, Cumming BG, Parker AJ (2002) Quantitative analysis of the responses of $\mathrm{V} 1$ neurons to horizontal disparity in dynamic random-dot stereograms. J Neurophysiol 87:191-208

Rockland KS (1985) Anatomical organisation of primary visual cortex (area 17) in the ferret. J Comp Neurol 241:225-236 
Shatz C (1977) A comparison of visual pathways in Boston and Midwestern siamese cats. J Comp Neurol 171:205-228

Skottun BC, De Valois RL, Grosof DH, Movshon JA, Albrecht DG, Bonds AB (1991) Classifying simple and complex cells on the basis of response modulation. Vision Res 31:1079-1086

Smith DC, Lorber R, Stanford LR, Loop MS (1980) Visual acuity following binocular deprivation in the cat. Brain Res 183:1-11

Stone J (1965) A quantitative analysis of the distribution of ganglion cells in the cat's retina. J Comp Neurol 124:337-352

Stone J, Rowe MH, Campion JE (1978) Retinal abnormalities in the Siamese cat. J Comp Neurol 180:773-782

Wen GY, Sturman JA, Shek JW (1985) A comparative study of the tapetum, retina and skull of the ferret, dog and cat. Lab Anim Sci $35: 200-210$
Wheatstone FRS (1838) Contributions to the physiology of vision. Part the first. On some remarkable, and hitherto unobserved phenomena of binocular vision. Philos Trans R Soc Lond 128:371394

Williams RW, Cavada C, Reinoso-Suarez F (1993) Rapid evolution of the visual system: a cellular assay of the retina and dorsal lateral geniculate nucleus of the Spanish wildcat and the domestic cat. J Neurosci 13:208-228

Wong-Riley M (1979) Changes in the visual system of monocularly sutured and enucleated cats demonstrable with cytochrome oxidase histochemistry. Brain Res 171:11-28 